\title{
Mechanisms of immunotherapeutic intervention by anti-CD40L (CD154) antibody in an animal model of multiple sclerosis
}

\author{
Laurence M. Howard, ${ }^{1}$ Amy J. Miga, ${ }^{2}$ Carol L. Vanderlugt, ${ }^{1}$ Mauro C. Dal Canto, ${ }^{3}$ \\ Jon D. Laman, ${ }^{4}$ Randolph J. Noelle, ${ }^{2}$ and Stephen D. Miller ${ }^{1}$
}

\begin{abstract}
${ }^{1}$ Department of Microbiology-Immunology, Northwestern University Medical School, Chicago, Illinois 60611, USA
${ }^{2}$ Department of Microbiology, Dartmouth University Medical School, Lebanon, New Hampshire 03756, USA

${ }^{3}$ Department of Pathology and the Interdepartmental Immunobiology Center, Northwestern University Medical School, Chicago, Illinois 60611, USA

${ }^{4}$ Department of Immunology, Erasmus University, Rotterdam, and Division of Immunological and Infectious Diseases, TNO, Prevention and Health, Leiden 2301 CE, the Netherlands.

Address correspondence and reprint requests to: Stephen D. Miller, Department of Microbiology-Immunology, Northwestern University Medical School, 303 East Chicago Avenue, Chicago, Illinois 60611, USA. Phone: (312) 503-7674; Fax: (312) 503-1339;

E-mail: s-d-miller@nwu.edu
\end{abstract}

Laurence M. Howard and Amy J. Miga contributed equally to this work.

Received for publication October 1, 1998, and accepted in revised form November 17, 1998.

\begin{abstract}
Relapsing experimental autoimmune encephalomyelitis (R-EAE) in the SJL mouse is a Th1-mediated autoimmune demyelinating disease model for human multiple sclerosis and is characterized by infiltration of the central nervous system (CNS) by Th1 cells and macrophages. Disease relapses are mediated by $T$ cells specific for endogenous myelin epitopes released during acute disease, reflecting a critical role for epitope spreading in the perpetuation of chronic central CNS pathology. We asked whether blockade of the CD40-CD154 (CD40L) costimulatory pathway could suppress relapses in mice with established REAE. Anti-CD154 antibody treatment at either the peak of acute disease or during remission effectively blocked clinical disease progression and CNS inflammation. This treatment blocked Th1 differentiation and effector function rather than expansion of myelin-specific $\mathrm{T}$ cells. Although T-cell proliferation and production of interleukin (IL)-2, IL-4, IL-5, and IL-10 were normal, antibody treatment severely inhibited interferon- $\gamma$ production, myelin peptide-specific delayed-type hypersensitivity responses, and induction of encephalitogenic effector cells. Anti-CD154 antibody treatment also impaired the expression of clinical disease in adoptive recipients of encephalitogenic T cells, suggesting that CD40-CD154 interactions may be involved in directing the CNS migration of these cells and/or in their effector ability to activate CNS macrophages/microglia. Thus, blockade of CD154-CD40 interactions is a promising immunotherapeutic strategy for treatment of ongoing $\mathrm{T}$ cell-mediated autoimmune diseases.
\end{abstract}

J. Clin. Invest. 103:281-290 (1999).

\section{Introduction}

Relapsing experimental autoimmune encephalomyelitis (R-EAE) is a Th1-mediated autoimmune demyelinating disease of the central nervous system (CNS), that serves as a useful model for multiple sclerosis (MS) (1). Induction of R-EAE in the susceptible SJL mouse strain by subcutaneous inoculation with the major immunodominant epitope of proteolipid protein (PLP139-151) in complete Freund's adjuvant (CFA) or by the adoptive transfer of PLP139-151-specific T cells results in a disease course characterized by a moderate to severe acute paralytic phase followed by remission and the subsequent development of spontaneous relapses (2). Relapsing disease episodes are accompanied by the development of T-cell responses to noninducing epitopes on the same or distinct myelin proteins secondary to acute CNS damage, a phenomenon termed epitope spreading $(3,4)$. In PLP139-151-induced R-EAE, the first clinical relapse is mediated predominantly by $\mathrm{T}$ cells specific for a secondary PLP epitope, PLP178-191 (4-6).

Regulation of ongoing R-EAE in the SJL/J mouse has been achieved by several immunoregulatory approaches including peptide-specific tolerance and blockade of costimulatory molecules involved in T-cell activation. We and others have shown that induction of peptidespecific tolerance by the intravenous injection of peptide-pulsed, ethylene carbadiimide (ECDI)-fixed antigen-presenting cells (APCs) blocks both induction and progression of R-EAE (7-9). This effect is mediated largely through the induction of anergy owing to the inability of the fixed APCs to express required costimulatory molecules necessary for effective T-cell induction (10). In support of the blockade of the B7/CD28 costimulatory pathway leading to antigen-specific unresponsiveness, a variety of studies have shown that direct interference with the B7/CD28 costimulatory pathway is an effective means of preventing induction of R-EAE $(11,12)$ and of treating ongoing disease $(6$, 13). In addition, the CD40-CD40L (CD154) ligand pair has been shown to be important for experimental autoimmune encephalomyelitis (EAE) induction (14), and blockade of this interaction using anti-CD154 
antibody inhibits early events in disease initiation (15). Blockade of CD154-CD40 interactions have also been shown to prevent the induction of other autoimmune models such as oophoritis (16), experimental autoimmune thyroiditis (17), lupus nephritis (18), collageninduced arthritis (19), and spontaneous autoimmune diabetes (20), indicating the tremendous potential of this immunoregulatory strategy in treating autoimmune disease. In addition, recent studies have demonstrated that blockade of this interaction also inhibits atherogenesis in hyperlipidemic mice (21).

CD40-CD154 interactions play multifunctional roles in the immune system. Although originally identified as a constitutive B-cell antigen, CD40 is expressed by many cells, including dendritic cells, macrophages, and astrocytes (22). CD154 (CD40L), the ligand for CD40, is transiently expressed primarily by activated CD4 T cells, although recently it has been identified on a subpopulation of activated B cells $(23,24)$. CD154 is upregulated by ligation of the T-cell receptor (TCR) and can be further increased by costimulatory events (24). CD40 ligation leads to the upregulation of the costimulatory molecules B7-1 (CD80) and B7-2 (CD86) on APCs, enhancing their ability to activate naive T-cells $(24,25)$. CD40-CD154 interactions are crucial for B cell activation and differentiation $(24,25)$ and for production of interleukin-12 (IL-12) by APCs, which biases CD4 T-cell responses toward Th1 (26).

While anti-CD154 has been shown to inhibit induction of EAE, the more relevant clinical potential of anti-CD154 therapy in treating ongoing EAE has yet to be evaluated. In this article, we evaluate the role of anti-CD154 in inhibiting ongoing disease and investigate the mechanisms by which inhibition occurs. We show for the first time that anti-CD154 treatment is an effective way of treating an established CD4 Th1-mediated autoimmune disease in that it rapidly ameliorated ongoing acute PLP139-151-induced R-EAE and, more importantly, inhibited expression of clinical relapses when administered during disease remission. Disease inhibition was associated with a dramatic decrease in immune cell infiltrates into the CNS and little or no demyelination. AntiCD154 therapy led to decreased lymph node T-cell expansion, but its effect was primarily associated with a dramatic inhibition of differentiation of Th1 responses to both the initiating and relapse-associated PLP epitopes.

\section{Methods}

Mice. Female SJL mice 5-6 weeks old were obtained from Harlan Sprague Dawley Inc. (Indianapolis, Indiana, USA) and NCI Laboratories (Frederick, Maryland, USA). Mice were housed under barrier conditions, and paralyzed mice were afforded easier access to food and water.

Peptides. PLP139-151 (HSLGKWLGHPDKF) and PLP178-191 (NTWTTCQSIAFPSK) were synthesized by the peptide facility at the University of North Carolina at Chapel Hill. Amino acid composition of these peptides was verified by mass spectrometry, and purity (>97\%) was confirmed by mass spectroscopy at the Michigan State University Biotechnology Center. PLP178-191 was insoluble in PBS and was dissolved in $0.035 \mathrm{M}$ acetic acid.

In vivo antibody treatment. Anti-CD154 (MR-1) was produced in ascites and purified by HPLC over a DEAE column. Normal hamster serum was purchased from Harlan Bioproducts for Science Inc. (Indianapolis, Indiana, USA) or Caltag Laboratories Inc.
(Burlingame, California, USA) and purified in the same manner. Mice were treated three to four times with $200 \mu \mathrm{g}$ of control hamster Ig or MR-1 antibody intraperitoneally in a total volume of $200 \mu \mathrm{l}$ administered every other day starting at either time of peptide inoculation, peak of acute phase, or when mice had entered remission from acute phase.

Induction of active and adoptive R-EAE. For actively induced REAE, mice were immunized subcutaneously with $100 \mu \mathrm{l}$ of a CFA emulsion containing $200 \mu \mathrm{g}$ of Mycobacterium tuberculosis $\mathrm{H} 37 \mathrm{Ra}$ (Difco Laboratories, Detroit, Michigan, USA) and $50 \mu \mathrm{g}$ of PLP139-151 distributed over three sites on the lateral hind flanks and dorsally. For adoptive transfer of R-EAE, female donor SJL/J mice (6-8 weeks old) were primed subcutaneously with $100 \mu \mathrm{g}$ of PLP139-151 in CFA distributed over four dorsal sites. Some mice received $250 \mu \mathrm{g}$ MR1 intraperitoneally on days 0,2 , 4, and 6 . Draining lymph nodes (axillary and inguinal) were harvested on day 8 and cultured in vitro in 24 -well plates $\left(8.0 \times 10^{6}\right.$ cells/well) with supplemented Roswell Park Memorial Institute medium (RPMI) (Sigma Chemical Co., St. Louis, Missouri, USA) and $70 \mu \mathrm{g} / \mathrm{ml}$ PLP. MR-1 $(25 \mu \mathrm{g} / \mathrm{ml})$ was added to some of the in vitro cultures. After 4 days in culture, the cells were harvested and centrifuged over a Ficoll gradient to remove debris. Recipient female SJL/J mice were intravenously injected with $1.25 \times 10^{6}, 2.5$ $\times 10^{6}$, or $5.0 \times 10^{6}$ cells in a final volume of $500 \mu$ l. Donors and recipients that were treated with anti-CD154 were given $250 \mu \mathrm{g}$ intraperitoneally three times a week.

Clinical evaluation. Mice were evaluated daily for clinical signs of disease for up to 40 days after inoculation. Clinical severity was assessed on a 0-5 scale as follows: grade 1, limp tail; grade 2, limp tail and hindlimb weakness (waddling gait); grade 3, partial hindlimb paralysis; grade 4 , complete hindlimb paralysis; and grade 5 , moribund. The data are plotted as the daily mean clinical score for all animals in a particular treatment group or as the relapse rate (total number of relapses in a group divided by the total number of mice in that group). A relapse was defined as a sustained increase of at least one full grade in clinical score after the animal had previously improved at least a full clinical score and had stabilized. Scores of asymptomatic mice ( score $=0$ ) were included in the calculation of the daily mean clinical score for each group.

Histology. Anesthetized mice were sacrificed by total body perfusion with chilled $3 \%$ glutaraldehyde in PBS, pH 7.3. Spinal cords were removed by dissection, cut into 1 -mm-thick coronal segments, postfixed in $1 \%$ osmium tetroxide, dehydrated, and embedded in Epon as described previously (27). Toluidine blue-stained sections from 10 segments per mouse were read blindly and scored as follows: \pm , mild inflammation without demyelination; $1+$, inflammation with focal demyelination; $2+$, inflammation with multiple foci of demyelination; $3+$, marked inflammation with bilateral, converging areas of demyelination; $4+$, extensive bilateral areas of demyelination and remyelination.

Delayed-type hypersensitivity responses. Delayed-type hypersensitivity (DTH) responses to PLP139-151 and to PLP178-191 were quantitated using a 24-h ear-swelling assay either directly after the acute phase of disease or during remission. Pre-challenge ear thickness was determined using a Mitutoyo model 7326 engineer's micrometer (Schlesinger's Tools, Brooklyn, New York, USA). DTH responses were elicited by injecting $10 \mu \mathrm{g}$ of peptide (in $10 \mu \mathrm{l}$ of saline) into the dorsal surface of the ear using a $100-\mu 1$ Hamilton syringe fitted with a 30 -gauge needle. Twenty-four hours after ear challenge, the increase in ear thickness over pre-challenge measurements was determined. Results are expressed in units of $10^{-4}$ in. \pm SEM.

In vitro T-cell proliferation assays. Spleen and lymph node cells were obtained from mice at various stages of disease progression, and dissociated cells were cultured in 96-well microtiter plates (Corning-Costar Corp., Acton, Massachusetts, USA) at a density of $5 \times 10^{5}$ viable cells per well in a total volume of 200 


\section{Figure 1}

Anti-CD154 inhibits clinical progression of established R-EAE. Mice were primed with PLP139-151/CFA as described in Methods. Mice were treated with either anti-CD154 or control hamster IgG at time of priming $(\boldsymbol{a})$, at the peak of acute disease $(\boldsymbol{b})$ (square symbols), or during the remission following recovery from acute disease (b) (triangular symbols). The data are expressed as the mean clinical score versus days after immunization for mice treated at the time of immunization (a), or as relapse rate for those mice treated during ongoing disease $(b)$. Data shown are representative of two similar experiments. $A b$, antibody; $R-E A E$, relapsing experimental autoimmune encephalomyelitis.
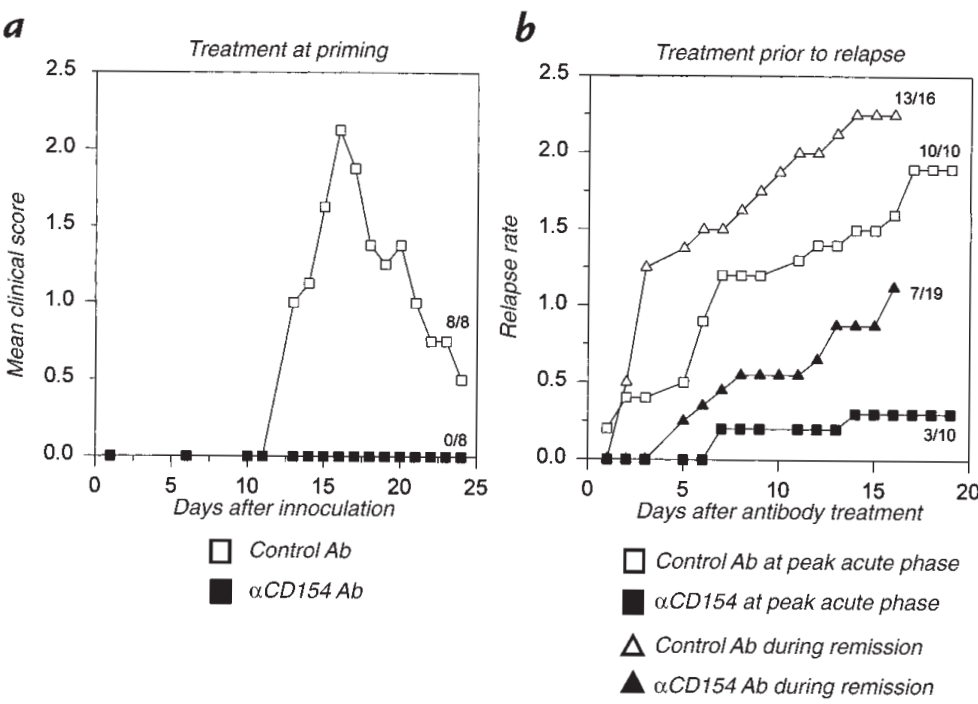

$\mu 1$ DME or RPMI-1640 (Sigma Chemical Co.) supplemented with $10 \%$ FCS (Sigma Chemical Co.), $100 \mathrm{U} / \mathrm{ml}$ penicillin (Life Technologies Inc., Gaithersburg, Maryland, USA), $100 \mu \mathrm{g} / \mathrm{ml}$ streptomycin (Life Technologies Inc.), and $2 \mathrm{mM}$ L-glutamine (Life Technologies Inc.). Cells were cultured at $37^{\circ} \mathrm{C}$ in $100 \%$ humidity and $5 \% \mathrm{CO}_{2}$ in the presence or absence (background) of varying concentrations of either PLP139-151 or PLP178-191. As a control, concanavalin A (Sigma Chemical Co.) was used at a concentration of $5 \mu \mathrm{g} / \mathrm{ml}$. Cultures were pulsed with $1 \mu \mathrm{Ci}$ of ${ }^{3} \mathrm{H}-\mathrm{TdR}$ (ICN Radiochemicals Inc., Irvine, California, USA) after $72 \mathrm{~h}$, harvested at $96 \mathrm{~h}$, and ${ }^{3} \mathrm{H}-\mathrm{TdR}$ uptake was detected using a Packard Topcount microplate scintillation counter (Packard Instrument Co., Meriden, Connecticut, USA). Results are expressed as mean of triplicate cultures \pm SEM.

Cytokine analysis. Draining lymph nodes (axillary and inguinal) and spleen cells from mice primed with PLP139$151 /$ CFA were removed on day 10 . A total of 200,000 cells/well were plated onto 96-well flat-bottom plates in supplemented DMEM and a range of PLP139-151 antigen concentrations. Supernatants were harvested on days 1-4 and analyzed for IL2 , IL-4, IL-5, IL-10, and interferon (IFN)- $\gamma$ by capture ELISA. ELISA reagents were purchased from Endogen Inc. (Woburn, Massachusetts, USA) and were used according to the manufacturer's protocol. Briefly, 96-well Nunc Maxisorb plates were coated overnight with anti-cytokine capture antibody followed by washing. Cytokine standards and samples were added to coated wells and were incubated at $20^{\circ} \mathrm{C}$ overnight and washed. Biotinylated anti-cytokine antibody was added, followed by washing and the addition of Streptavidin-HRP conjugate. DACO TMB (DAKO Corp., Carpinteria, California, USA) liquid substrate system was used. The plates were read at $450 \mathrm{~nm}$.

Statistical analyses. Comparison of the percentage of animals showing clinical disease and/or relapses between any two groups of mice were analyzed by $\chi^{2}$ using Fisher's exact test. Comparisons of DTH responses and lymphoid cell recoveries were analyzed by Student's $t$ test. $P<0.05$ was considered significant.

\section{Results}

Anti-CD154 therapy inhibits clinical progression of established $R$ EAE. Previous studies have indicated that CD40-CD154 interactions are important for the development of acute EAE in mice, because disease is blocked in either CD154deficient mice (14) or mice treated with anti-CD154 mon- oclonal antibody (MAB) at the time of disease initiation (15). We wanted to confirm these results and to investigate the more clinically relevant ability of anti-CD154 MAB therapy to treat ongoing R-EAE. In accordance with previous findings, anti-CD154 administered during at the time of PLP139-151 priming totally inhibited the development of acute disease (Fig. 1a). Delay of treatment until mice reached the peak of acute disease resulted in a marked reduction in the relapse rate, with fewer mice exhibiting clinical signs of relapse in the CD154-treated group compared with the controls $(3 / 10[30 \%]$ relapses vs. $10 / 10$ $[100 \%]$ in control mice; $P<0.01)$ and a delay in onset of relapse for those mice exhibiting clinical signs (Fig. 1b). In addition, relapses were short-lived in CD154-treated mice compared with control mice, most of which relapsed more than once during the observation period. The mean clinical disease score for relapsing mice (excluding mice that did not relapse) was also markedly lower for the antiCD154-treated group $(0.7 \pm 0.6)$ compared with the control group (1.8 \pm 0.7$)$. The acute-phase disease profile was identical in each treatment group. In each group, 10 of 10 mice developed acute clinical disease, with onset at day 12 and disease peak on day 16 (mean clinical scores: $2.2 \pm 0.9$ for control group and $2.2 \pm 0.6$ for CD154-treated mice).

Delay of treatment until mice reached remission was nearly as effective as when treatment was initiated at the peak of acute phase (Fig. 1b). The relapse rate for antiCD154-treated mice was significantly reduced compared with that for control mice (7/19 [37\%] relapses vs. 13/16 $[81 \%]$ in control mice; $P<0.02)$. Again, onset of relapses was delayed in anti-CD154-treated mice, and disease severity of mice that relapsed in the treated group was markedly lower than that of their controls. Collectively, these results indicated that anti-CD154 therapy provides a highly effective way of treating ongoing R-EAE when administered either at the peak of acute disease or during early remission.

Diminished CNS infiltration of immune cells and demyelinating lesions in mice treated with anti-CD154 during ongoing disease. Histologic examination of spinal cord sections was carried out to determine whether the diminished clinical disease 
in anti-CD154-treated mice correlated with reduced CNS infiltration and demyelination. Antibody treatment at the time of priming prevented the appearance of infiltrates and demyelination in the spinal cords of mice examined after recovery from acute disease (compare Fig. $2, b$ with $a$ ). Spinal cords from control Ig-treated mice that had undergone at least one disease relapse exhibited markedly more extensive infiltrates and extensive demyelination accompanied by glial scarring when compared with mice examined after the acute phase of disease (compare Fig. 2, $c$ with a). In contrast, mice treated with anti-CD154 at the peak of acute disease showed few immune cell infiltrates, little or no demyelination, and no scarring (Fig. $2 d$ ). A semiquantitative comparison of the histology is summarized in Table 1 . There were few immune cell infiltrates into the CNS of anti-CD154-treated mice, whether treatment was initiated at the time of priming or at the peak of the acute phase of disease. This suggests that antiCD154 may act in part by either preventing activated myelin-specific $T$ cells and/or macrophages from entering the CNS or from being retained in the target organ.

Myelin-specific DTH reactivity in anti-CD154-treated mice. To gain an understanding of the mechanisms by which anti-CD154 therapy led to amelioration of ongoing disease, we assessed T-cell responses to both the initiating PLP139-151 and the relapse-associated PLP178-191 epi- topes by DTH, T-cell proliferation, and cytokine production assays. DTH responses measured during remission in mice treated either at the time of inoculation or at the peak of acute disease are shown in Fig. 3. DTH responses to the PLP139-151 priming epitope were significantly reduced in the group treated with anti-CD154 at disease initiation compared with those of hamster Ig-treated controls (Fig. $3 a$, left; $P<0.05$ ). Hamster Ig-treated controls demonstrated a significant DTH response to the relapseassociated PLP178-191 epitope, but anti-CD154-treated mice exhibited no significant response (Fig. $3 a$, right). A similar pattern was observed in mice treated at the peak of acute disease in that anti-CD154 treatment significantly
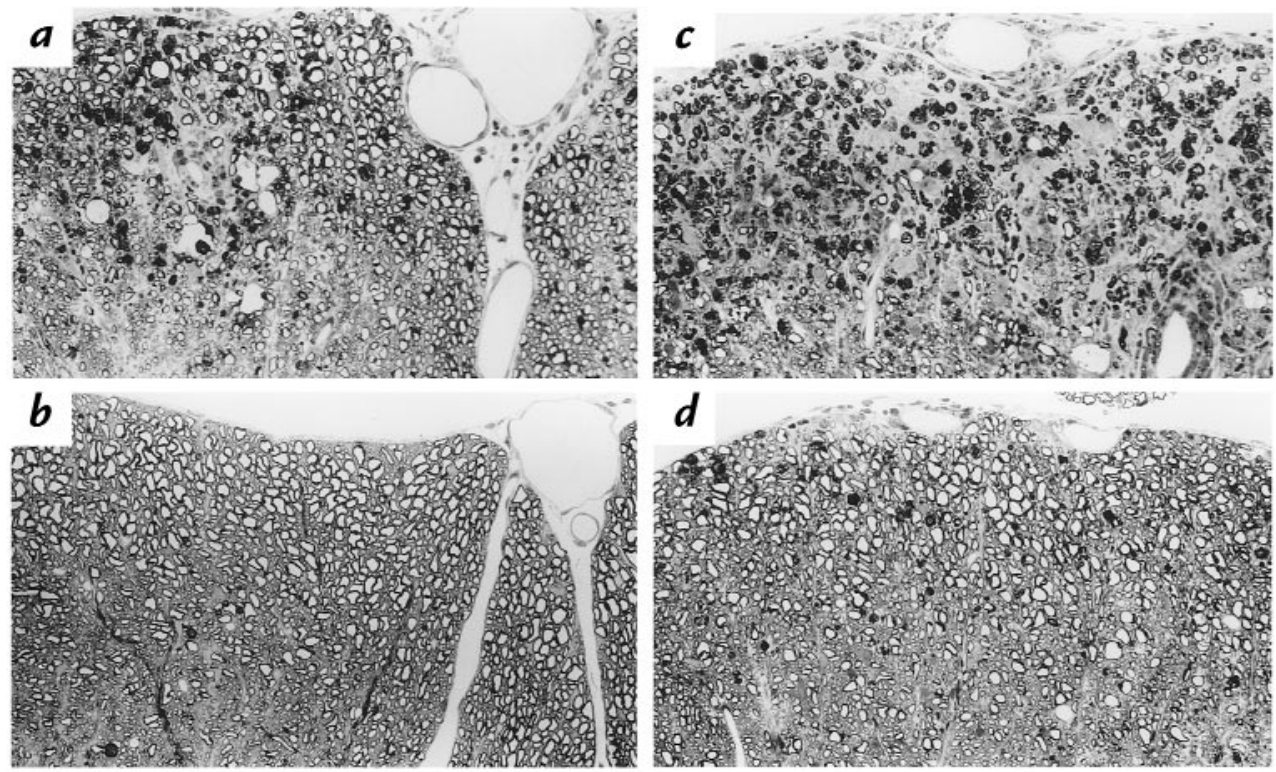

\section{Figure 2}

Effect of anti-CD154 treatment on CNS histology. Lumbar spinal cord sections from representative animals of the treatment groups described in Fig. 1 were examined for CNS histopathology. (a) Section of spinal cord from a mouse treated with control Ig at the time of priming and sacrificed on day 25. (b) Section of spinal cord from a mouse treated with anti-CD154 at the time of priming and sacrificed on day 25. (c) Section of spinal cord from a mouse treated with control Ig at the peak of acute disease and sacrificed on day 40 . (d) Section of spinal cord from a mouse treated with antiCD154 at the peak of acute disease and sacrificed on day 40. Details of the degree of inflammation and demyelination are given in Table 1. Sections shown in $a-d$ are $1-\mu \mathrm{m}$-thick Epon-embedded sections stained with toluidine blue. $\times 220$. CNS, central nervous system. 
inhibited DTH to both the initiating (Fig. 3b, left) and relapse-associated (Fig. 3b, right) epitopes. Thus, antiCD154 treatment of animals with ongoing R-EAE was significantly, but not totally, effective in inhibiting Th1 responsiveness and epitope spreading. This suggests that anti-CD154 acts, at least in part, by inhibiting the effector function of myelin-specific Th1 responses. Diminished in vivo Th1 cell function could occur by either specifically blocking expansion of myelin-specific Th1 responses and/or by inhibiting Th1 differentiation.

Myelin-specific T cell-proliferative and cytokine responses in anti-CD154-treated mice. To examine the potential effects of anti-CD154 treatment on Th1 expansion and/or differentiation, we assessed the level of myelin-specific T-cell proliferation and cytokine production in treated mice. Anti-CD154 treatment led to a $60 \%$ reduction in recovery of lymph node lymphocytes, but not splenic lymphocytes, in mice treated at the time of priming (Fig. 4; $P<$ $0.05)$. A similar reduction in lymph node cell numbers was seen in mice treated at peak of acute phase when cell numbers were assessed 10 days later (data not shown). The decreased recovery of lymph node lymphocytes in mice treated with anti-CD154 during priming was paralleled by a significant decrease in lymph node T cell-proliferative responses to the inducing PLP139-151 epitope when compared with controls (Fig. 4a). However, the splenic T cell-proliferative response to PLP139-151 was significantly higher in anti-CD154-treated mice 10 days after priming. No significant responses to the relapseassociated PLP178-191 epitope were observed in the lymph nodes or spleens of either control or antiCD154-treated mice (Fig. 4b), which is to be expected, as these animals had not yet undergone acute disease. A similar pattern of results was observed in mice treated at peak of acute disease. PLP139-151-specific proliferative responses of lymph node cells harvested when the mice reached remission (day 25 after immunization) was also significantly diminished in anti-CD154-treated mice compared with controls (Fig. 4c), whereas splenic responses were not suppressed. Noticeably, in both cases, levels of PLP139-151-specific proliferation of splenic and lymph node cells were reduced compared with the levels observed on day 10 after immunization (Fig. 4a). During remission, significant dose-dependent proliferative responses to the relapse-associated PLP178-191 epitope were observed in both spleen and lymph node cells of control mice (Fig. 4d). PLP178-191-specific responses were markedly reduced in the lymph nodes and increased in the spleens of anti-CD154-treated mice compared with controls. Because lymph node cell recovery was significantly lower from anti-CD154-treated compared with control mice, the diminished proliferative responses to both epitopes observed in this population could have been due to diminished numbers of $\mathrm{T}$ cells. This is unlikely, however, because concanavalin A-induced proliferation of lymph node $\mathrm{T}$ cells was comparable in antiCD154-treated and control mice (data not shown). Collectively, although myelin epitope-specific T-cell proliferative responses were reduced in the lymph nodes of antibody-treated mice, the degree of suppression is unlikely to account for the very marked effects of anti$\mathrm{CD} 154$ on clinical disease in light of the enhanced
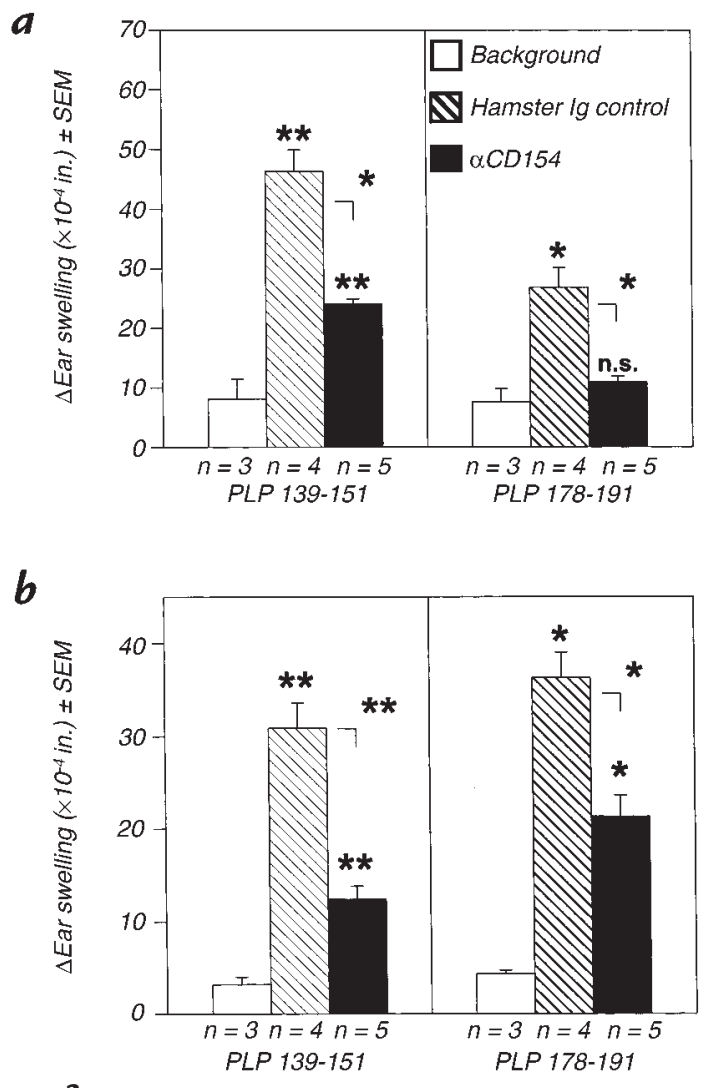

Figure 3

Anti-CD154 treatment inhibits DTH responses to both the initiating and relapse-associated myelin epitopes. DTH responses to both the initiating PLP139-151 peptide and to the relapse-associated PLP178-191 peptide were evaluated in mice treated with either control Ig or anti-CD154 at the time of priming $(\boldsymbol{a})$ or at the peak of acute disease $(\boldsymbol{b})$. Data represent mean \pm SD of the change in ear thickness $24 \mathrm{~h}$ after ear challenge with 10 $\mu \mathrm{g}$ of peptide. The numbers of mice in each group are shown below each bar. Data are representative of two experiments with similar results. DTH responses significantly less than those of control Ig-treated mice: ${ }^{*} P<$ $\left.0.05 ;{ }^{*} P<0.01\right)$. Results shown above each bar show statistical comparisons to background ear-swelling levels, i.e., levels in naive mice challenged with each peptide. DTH, delayed-type hypersensitivity.

responses observed in the splenic T-cell compartment. Anti-CD154 treatment blocks the differentiation of PLP139151-specific Th1 cells. The proliferative data suggest that anti-CD154 may partially interfere with the clonal expansion of PLP-specific T cells. To determine the effects of anti-CD154 treatment on Th1 differentiation, we assessed the ability of PLP-responsive T cells to produce both Th1 (IFN- $\gamma$ and IL-2)- and Th2 (IL-4, IL-5, and IL-10)-type cytokines. Secondary in vitro stimulation of PLP139-151-specific T cells, derived from the spleens of mice treated with anti-CD154 at time of priming, demonstrated control levels of IL-2 production (Fig. 5a); however, IFN- $\gamma$ production was almost completely suppressed (Fig. 5c). Lymph node-derived cells also exhibited significantly reduced levels of IFN- $\gamma$ production (Fig. $5 d$ ); however, unlike splenic T cells, IL-2 production was also somewhat reduced (Fig. 5b). PLP139-151-induced IL-4 and IL-5 production was also assessed, but only minimal levels of secretion of these cytokines were observed in all cultures (data not shown). Similarly, low, 

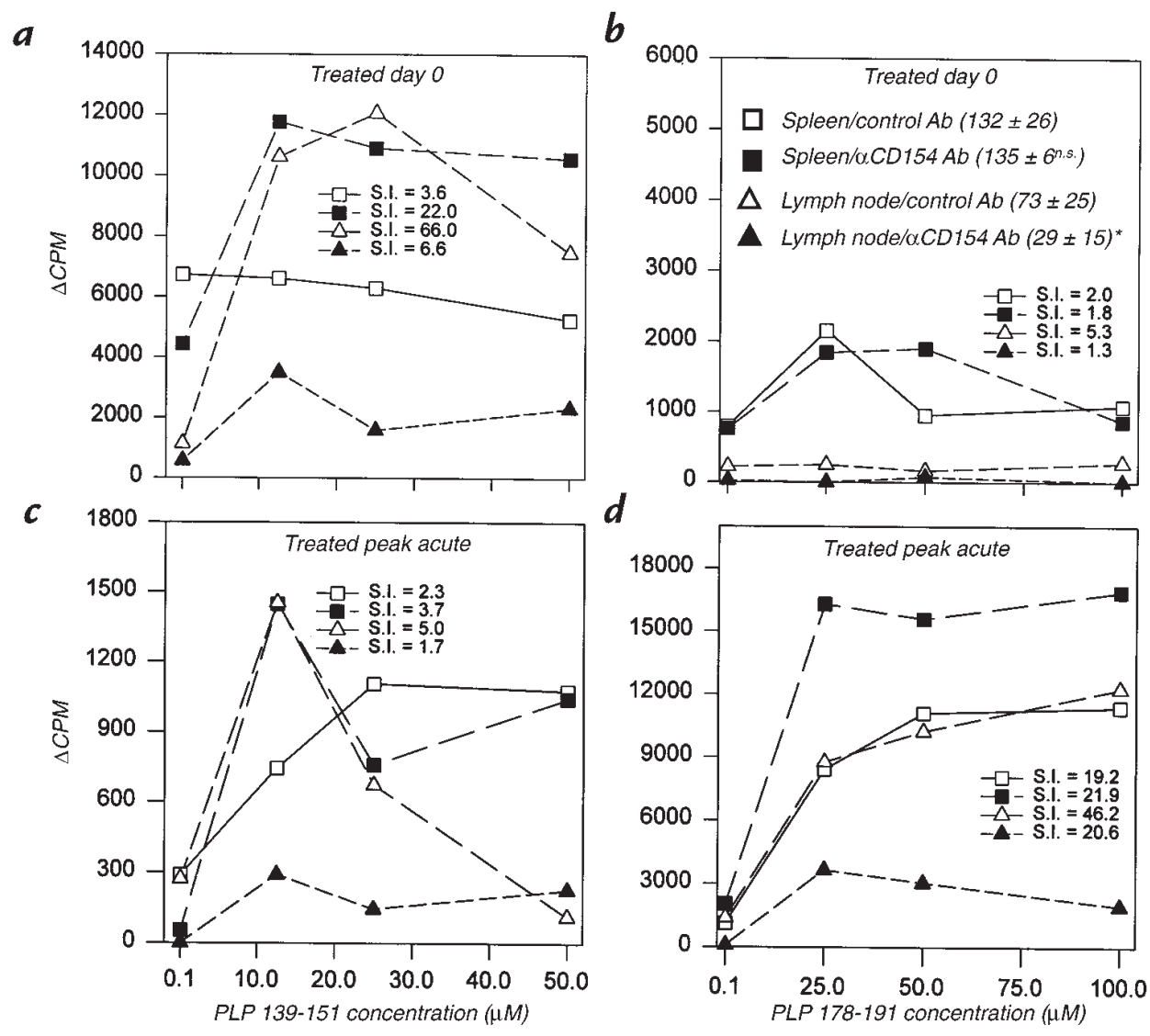

Figure 4

Myelin epitope-specific in vitro proliferative responses of spleen and lymph node cells from mice treated with anti-CD154 during disease induction or at the peak of acute disease. Splenic and lymph node lymphocytes from three mice each treated with control Ig or anti-CD154 either at the time of priming were harvested at day 10 after immunization $(\boldsymbol{a}$ and $\boldsymbol{b})$ or at the peak of acute disease were harvested at day 25 after immunization ( $\boldsymbol{c}$ and d). A total of $5 \times 10^{5}$ viable cells per well were cultured with varying concentrations of PLP139-151 or PLP178-191 for 4 days. Cultures were pulsed with ${ }^{3} \mathrm{H}-\mathrm{TdR} 18-24 \mathrm{~h}$ before harvest. Data are presented as $\triangle \mathrm{CPM}\left({ }^{3} \mathrm{H}-\mathrm{TdR}\right.$ incorporation in cultures containing peptide antigen - ${ }^{3} \mathrm{H}-\mathrm{TdR}$ incorporation in cultures containing medium). Data shown are representative of two experiments. Mean \pm SD of viable lymphocytes recovered from each treatment group (minimum of three mice each from three separate experiments) is shown in the legend $(* P<0.05)$. Stimulation indices $(S . I$.) are shown for each treatment group at the concentration of peptide that stimulated optimal proliferation. An S.I. $\geq 3$ is considered significant.

but comparable, levels of IL-10 secretion were observed in both control and anti-CD154-treated mice (Fig. 5, e and $f$ ). The lack of IFN- $\gamma$ production by splenic $\mathrm{T}$ cells from anti-CD154-treated mice is particularly remarkable, because this population exhibited enhanced proliferative capacity (Fig. $4 a$ ) and produced control levels of IL-2 (Fig. 5a). These results are consistent with the ability of anti-CD154 to inhibit Th1 differentiation in other autoimmune models $(17,20,28)$, as well as in models of graft-versus-host disease and graft rejection $(29,30)$ and with our observation that anti-CD154 treatment significantly inhibited development of DTH responses (Fig. 3).

Anti-CD154 treatment of donor mice inbibits the generation of encephalitogenic $T$ cells. To gain further understanding of how anti-CD154 treatment prevents EAE, the effect of anti-CD154 treatment on adoptive R-EAE was studied. SJL/J mice were immunized with PLP139-151/CFA and treated with anti-CD154 or hamster Ig on days $0,2,4$, and 6. On day 8 , the draining lymph nodes were removed and activated in vitro with PLP139-151 for four days, and varying numbers of viable cells $\left(2.5 \times 10^{6}-10^{7}\right)$ were adoptively transferred into naive SJL/J recipients. Treatment of donor mice with anti-CD154 reduced the capacity of PLP139-151-specific T cells to transfer disease as judged by a reduction in disease severity and/or onset. Inhibition was most evident when limiting numbers of donor cells $\left(2.5 \times 10^{6}\right.$ to $5 \times 10^{6}$ cells/mouse; Fig. $6, b$ and $\left.c\right)$ from antiCD154-treated mice were transferred. Inclusion of antiCD154 during the in vitro culture only did not decrease the encephalitogenicity of the donor T cells (Fig. 6b). However, complete blockade of disease transfer was observed when the donor $\mathrm{T}$ cells were derived from mice treated with anti-CD154 and $\alpha$-CD154 was included during the in vitro activation culture and cells transferred to antiCD154-treated recipients. Therefore, anti-CD154 treatment blocks the generation of encephalitogenic $\mathrm{T}$ cells consistent with its suppressive effects on development of clinical disease (Fig. 1) and differentiation of myelin epitope-specific Th1 cells (Figs. 3 and 5).

Anti-CD154 treatment of recipients reduces the onset and severity of R-EAE. The fact that treatment of mice at the peak of acute disease or during remission ameliorated relapsing disease suggests that anti-CD154 can interfere with disease development even after the expansion and differentiation 
of encephalitogenic T cells. To assess directly the effects of anti-CD154 on the effector phase of disease, graded numbers $\left(1.25 \times 10^{6}\right.$ to $\left.5 \times 10^{6}\right)$ of PLP139-151-primed T cells were adoptively transferred into naive recipients who were subsequently treated with anti-CD154 or control Ig. AntiCD154 treatment significantly reduced disease severity even upon the transfer of the highest numbers of primed T cells (Fig. $7 a ; 5 \times 10^{6} /$ mouse). More profound inhibition of disease transfer was observed in treated recipients receiving lower numbers of transferred cells (Fig. 7, $b$ and $c$ ). Blockade of encephalitogenic T-cell function may be due to either interference with CNS homing of the activated encephalitogenic $\mathrm{T}$ cells or direct interference with other effector functions (i.e., the ability to produce/secrete proinflammatory cytokines), or both.

\section{Discussion}

CD40-CD40L (CD154) interaction plays multiple important roles in regulating both humoral and cell-mediated immune responses. Significant to its potential as an immunotherapeutic agent for the treatment of autoimmune diseases, anti-CD154 has been shown previously to block the spontaneous development of pathogenic autoantibodies in lupus-prone mice $(18,31)$ and to inhibit the induction of experimental collagen-induced arthritis (19) and trinitrobenzene sulphonic acid (TNBS)induced colitis (28). CD40-CD40L interactions have also been implicated in the induction of R-EAE. CD154 knockout mice, expressing an MBP-specific transgenic TCR, fail to develop clinical disease (14), and antibody to CD154 has been shown to inhibit EAE induction (15).

At present, there is little information on the mechanism(s) whereby anti-CD154 inhibits EAE induction, and none on its therapeutic potential for treating ongoing $\mathrm{T}$ cell-mediated autoimmune diseases such as REAE. Such knowledge is extremely relevant to the potential application of this therapy in human multiple sclerosis and other autoimmune diseases. In this article, we address both of these questions and show that CD40/CD154 interactions are critical for both the initiation and progression of R-EAE. Anti-CD154 treatment effectively inhibits R-EAE when administered both at time of disease induction, confirming earlier studies (15), and, more importantly, during ongoing R-EAE. In addition, we show that anti-CD154-induced inhibition of clinical disease is associated with differential regulation of lymph node and splenic responses to self myelin peptides, resulting in reduced activation of Th1-type responses and inhibition of the effector function of differentiated encephalitogenic T cells.

Treatment of ongoing R-EAE with anti-CD154 was more or less equally effective when given either early (at peak of acute disease) or later when mice were in remission following the acute disease incident (Fig. 1b). Treatment resulted in a significant reduction in the percentage of animals displaying disease relapses and a delay in the onset and clinical severity in those mice that did relapse. Treatment at either time during ongoing disease also resulted in a profound reduction of inflammation and demyelination in the CNS (Fig. 2). The ameliorating effects of anti-CD154 therapy could occur at multiple levels. Relapses in PLP139-151-induced R-EAE in the SJL
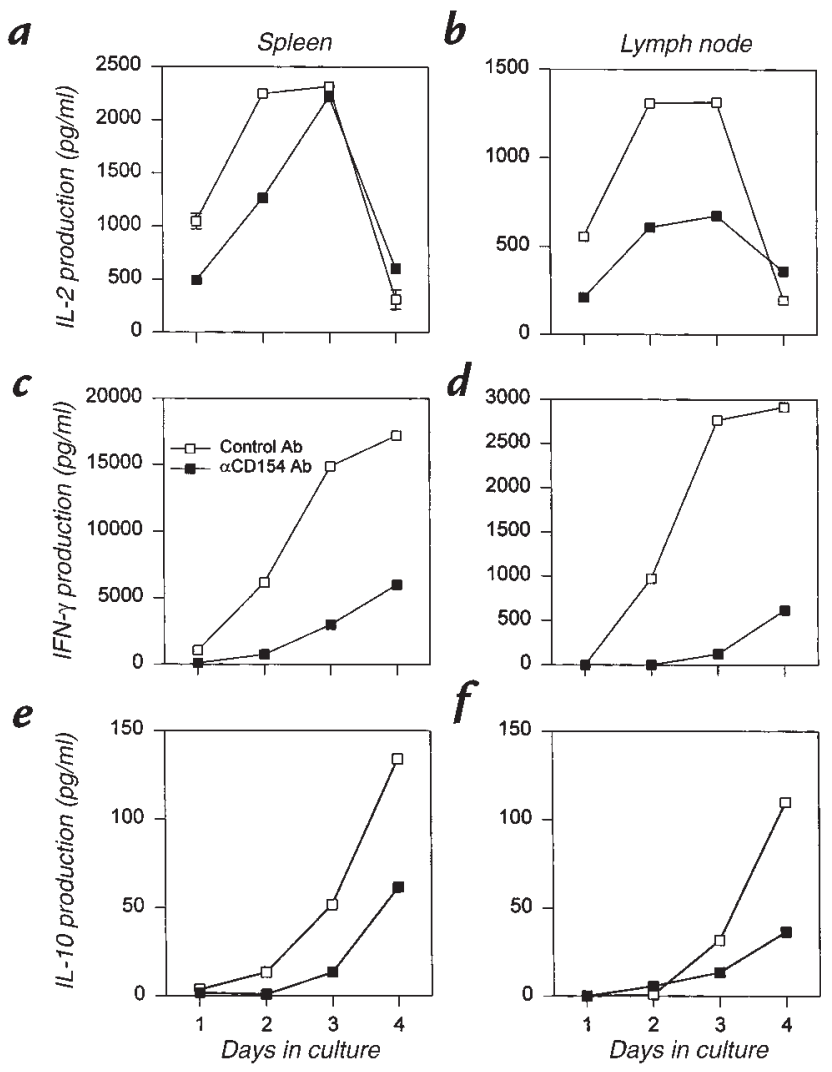

$f$

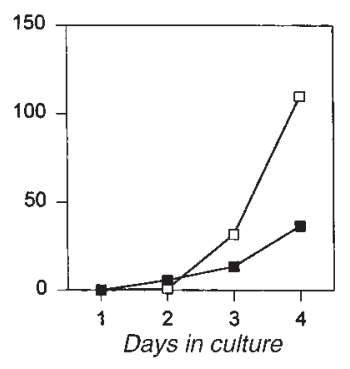

Figure 5

Anti-CD154 treatment blocks the differentiation of PLP139-151-specific, IFN- $\gamma$-producing Th1 cells. Splenic and lymph node lymphocytes from three mice each treated at time of priming with either control Ig or antiCD154 were harvested at day 10 after immunization. A total of $2 \times 10^{5}$ viable cells/well were cultured with an optimal dose of PLP139-151 (25 $\mu \mathrm{M})$. Culture supernatants were harvested each day and analyzed for IL2, IFN- $\gamma$, and IL-10 by ELISA, as described in Methods. Data shown are representative of two experiments. In all cases, the SD was less than $10 \%$ of the values. IFN, interferon; IL, interleukin.

mouse are mediated largely by epitope spreading (4), wherein T cells specific for the endogenous PLP178 epitope are primed de novo in response to myelin damage mediated by $T$ cells specific for the initiating epitope. Thus, anti-CD154 may act in part by inhibiting the expansion and/or differentiation of Th1 cells specific for relapse-associated epitopes. Blockade of CD40/CD154 interactions could also directly interfere with the effector functions of differentiated Th1 effector cells. This could occur via direct effects on the production of proinflammatory cytokines or by interfering with the ability of encephalitogenic T cells to transmigrate into the CNS and/or recruit and activate macrophages. In contrast to R-EAE, in the NOD mouse, CD40/CD154 blockade inhibits development of diabetes only when administered to young mice (4 weeks of age), but not in older mice (9-10 weeks of age), when significant autoimmune disease has already developed (20). This may reflect the difference in a chronic progressive diabetes model versus the relapsing EAE model, perhaps associated with the transient nature of CD40/CD154 interaction in initiating responses to relapse-associated epitopes. 
The possibility that anti-CD154 may directly block transmigration of encephalitogenic $\mathrm{T}$ cells across the blood-brain barrier is suggested by the fact that adoptive transfer of R-EAE was significantly, but not completely, inhibited by anti-CD154 treatment of recipients of activated PLP139-151-specific T cells (Figs. 6 and 7). CD40 ligation has been shown to activate endothelial cells upregulating expression of adhesion molecules (32) and production of proinflammatory cytokines (33) that can promote extravasation of lymphocytes. Although human endothelial cells have been shown to express functional CD40 $(24,32)$, our preliminary experiments examining CD40 and CD40L expression on mouse cerebrovascular endothelial cells (CVEs) in vivo and on cloned CVEs in vitro have failed to detect significant

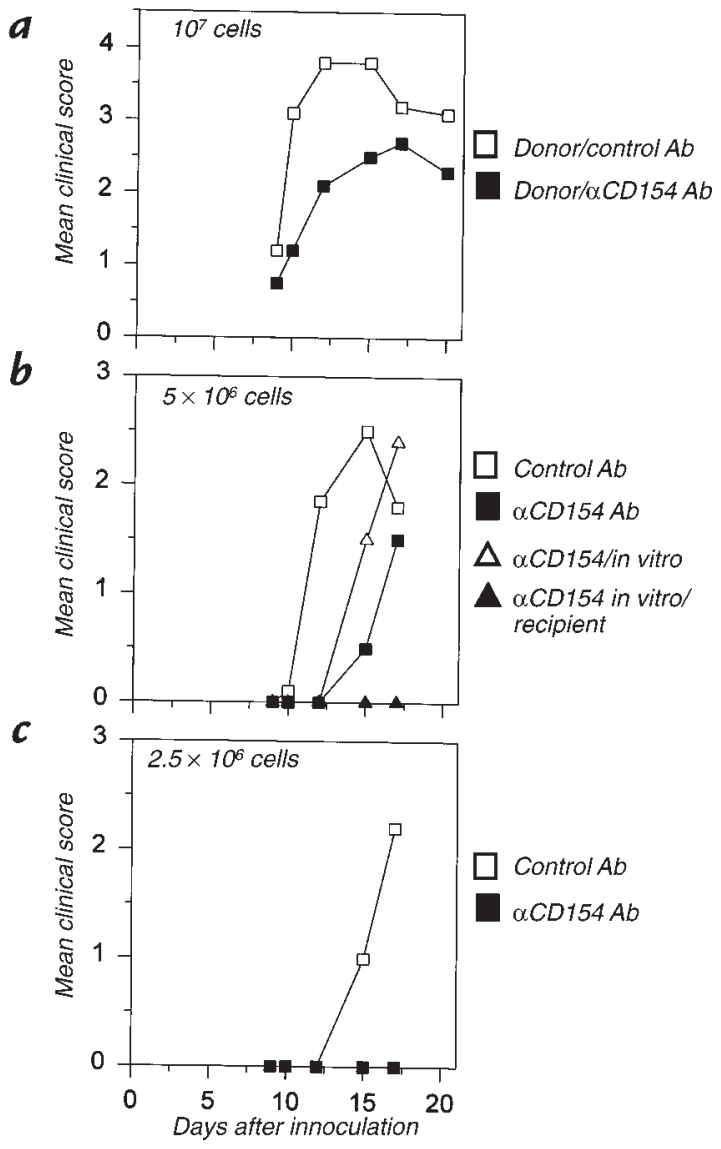

\section{Figure 6}

Anti-CD154 treatment of donor mice diminishes the capacity of PLP139151-primed T cells to transfer R-EAE. Female SJL/J mice were primed with PLP139-151/CFA. These donor mice treated with either $250 \mu \mathrm{g}$ of control Ig (open squares) or anti-CD154 (closed squares) on days $0,2,4$, and 6. Draining lymph nodes were harvested on day 8 and cultured with 70 $\mu \mathrm{g} / \mathrm{ml}$ PLP139-151 in vitro. After 4 days in culture, naive female SJL/J recipient mice were injected intravenously with $10^{7}(\boldsymbol{a}), 5 \times 10^{6}(\boldsymbol{b})$, or 2.5 $\times 10^{6}(\boldsymbol{c})$ viable cells. In $b$, some mice received cells from antiCD154-treated donors that were also treated with $25 \mu \mathrm{g} / \mathrm{ml}$ of antiCD154 in vitro (open triangles). In addition, some mice received cells from anti-CD154-treated donors that were exposed to anti-CD154 during the in vitro activation culture and transferred into anti-CD154 treated recipients (closed triangles). Recipients treated with anti-CD154 were injected with $250 \mu \mathrm{g}$ intraperitoneally three times a week. Mice were examined for disease severity and scored as described in Methods. expression of either molecule (Gordon, K.B., et al., manuscript in preparation). Thus, it appears unlikely that anti-CD154 directly obstructed T-cell migration into the CNS by blocking $\mathrm{T}$ cell-endothelial cell interactions, but we are continuing to examine this possibility using cell tracking studies employing the transfer of encephalitogenic T cells from SJL Thy1.1-congenic mice into naive SJL Thy1.2-bearing recipients. The in vivo effect on adoptive R-EAE more likely lies in the inhibition of T cell/APC interactions in the CNS of the recipient animal resulting in the downregulation of effector inflammatory responses. This could occur by blocking delivery of costimulatory signals to the encephalitogenic $T$ cells required for production of chemokines and/or proinflammatory cytokines, and/or by inhibiting CD40 signaling of CNSresident macrophages/microglia and astrocytes, preventing the production of inflammatory mediators, e.g., NO and matrix metalloproteinases (34-36).

Regarding the ability of anti-CD154 therapy to prevent the expansion and/or differentiation of myelin epitope-specific $\mathrm{T}$ cells, our results suggest that its major effect lies at the level of inhibiting the differentiation of Th1 cells specific for both the initiating and relapse-associated myelin epitopes. Using thymidine incorporation in both the spleens and draining lymph nodes of PLP139151-primed mice as a measure of T-cell expansion, our results suggest that the effects of anti-CD154 treatment were minimal. We observed an interesting dichotomy in that the lymphoid cell numbers and myelin epitope-specific proliferative responses were significantly diminished in the lymph nodes of anti-CD154-treated mice, but this was apparently balanced by a concomitant increase in splenic T-cell responses (Figs. 4 and 5). One possible explanation may lie in the fact that splenic APCs from autoimmune-prone SJL mice express higher constitutive levels of cell surface expression of CD80/CD86 costimulatory molecules than do lymph node APCs (37) and may thus be less sensitive to the inhibitory effects of antiCD154 on CD40-mediated upregulation of CD80/CD86 $(14,24,25,38,39)$ and IL-12 $(26,28,40,41)$ in APCs. Other recent studies have observed a similar reduction of T-cell expansion by anti-CD154 in the lymph nodes in a TCR transgenic mouse system (42), as well as in other EAE-susceptible mice (43), supporting the observations in this study. The differential effects on antigen-specific proliferative responses in the lymph nodes versus spleens of anti-CD154-treated mice may thus reflect diminished priming of T-cell precursors in the lymph node; migration of the precursor cells to the spleen, where they may become activated; and/or a switch to Th2-type response, through inhibition of IL-12 production by absence of CD40 ligation on the APCs. However, it should be noted that the latter possibility is apparently not supported by our failure to demonstrate IL-4, IL-5, or increased IL-10 production by either splenic or lymph node T cells from anti-CD154-treated mice (Fig. 6). Alternatively, the reduced lymph node response observed in antiCD154-treated mice may reflect a disruption of the architecture of the lymph nodes preventing efficient $\mathrm{T}$ cell expansion or retention.

Separate from its potential effects on T-cell expansion, the net result of anti-CD154 treatment is clearly a reduc- 
tion in Th1 differentiation and effector function. This is suggested by a number of pieces of experimental data. First, DTH responsiveness, a measure of in vivo generation of Th1 cells (44), to both the initiating and relapseassociated PLP epitopes is significantly reduced in antiCD154-treated mice (Fig. 3). Second, while in vitro production of IL-2 by myelin-specific $\mathrm{T}$ cells was largely unaffected, we observed dramatically reduced levels of IFN- $\gamma$ production (reflecting diminished Th1 responses) by both lymph node and splenic $T$ cells (Fig. 6), also suggesting that anti-CD154 blocked T-cell Th1 differentiation. This pattern of cytokine production suggests that $\mathrm{T}$ cells expanded in anti-CD154-treated mice possessed Th0 characteristics. Third, our results indicate that expansion of PLP139-151-specific encephalitogenic T cells in anti-CD154-treated donors was decreased in that these cells were less effective in transferring disease to naive SJL recipients (Fig. 7). By all of these criteria, antiCD154 therapy appears to have a major downregulatory effect on Th1 differentiation. Given that effective inhibition of disease transfer only occurred when recipient mice were also treated, it appears that anti-CD154 not only inhibits differentiation of encephalitogenic Th1 cells but may also interfere with their homing and/or effector functions as already discussed here.

In summary, these results verify the immunotherapeutic potential of anti-CD154 for treatment of established $\mathrm{T}$ cell-mediated autoimmune diseases. Experiments addressing the mechanisms accounting for the amelioration of ongoing R-EAE indicate a profound effect of CD40-CD154 blockade on the differentiation and effector functions of autospecific Th1 cells. Further characterization of lymph node and splenic cell populations in anti-CD154-treated mice should provide significant information as to the exact molecular events involved in disease regulation.

\section{Acknowledgments}

This work was supported by US Public Health Service/National Institutes of Health research grants NS34819 (to S.D. Miller), NS30871 (to S.D. Miller), AI37075 (to R.J. Noelle), and NS13011 (to M.C. Dal Canto), and by National Multiple Sclerosis Society grants RG2275 (to S.D. Miller), RG2893 (to M.C. Dal Canto), and 5-38229 (to R.J. Noelle).

1. Brown, A.M., and McFarlin, D.E. 1981. Relapsing experimental allergic encephalomyelitis in the SJL/J mouse. Lab. Invest. 45:278-284

2. McRae, B.L., Kennedy, M.K., Tan, L.J., Dal Canto, M.C., and Miller, S.D. 1992. Induction of active and adoptive chronic-relapsing experimental autoimmune encephalomyelitis (EAE) using an encephalitogenic epitope of proteolipid protein. J. Neuroimmunol. 38:229-240.

3. Lehmann, P.V., Sercarz, E.E., Forsthuber, T., Dayan, C.M., and Gammon, G. 1993. Determinant spreading and the dynamics of the autoimmune T-cell repertoire. Immunol. Today. 14:203-208.

4. McRae, B.L., Vanderlugt, C.L., Dal Canto, M.C., and Miller, S.D. 1995 Functional evidence for epitope spreading in the relapsing pathology of EAE in the SJL/J mouse. J. Exp. Med. 182:75-85.

5. Vanderlugt, C.L., and Miller, S.D. 1996. Epitope spreading. Curr. Opin. Immunol. 8:831-836.

6. Vanderlugt, C.L., Karandikar, N.J., Bluestone, J.A., and Miller, S.D. 1998. The functional significance of epitope spreading and its regulation by costimulatory interactions. Immunol. Rev. 164:63-72.

7. Kennedy, M.K., et al. 1990. Inhibition of murine relapsing experimental autoimmune encephalomyelitis by immune tolerance to proteolipid protein and its encephalitogenic peptides. J. Immunol. 144:909-915.

8. Kennedy, M.K., Tan, L.J., Dal Canto, M.C., and Miller, S.D. 1990. Regulation of the effector stages of experimental autoimmune encephalomyelitis via neuroantigen-specific tolerance induction. J. Immunol. 145:117-126.

9. Santambrogio, L., et al. 1995. Tolerogenic forms of auto-antigens and

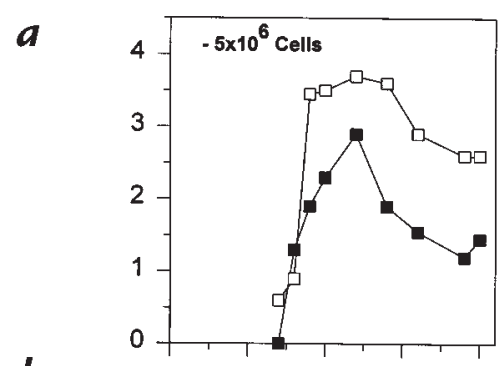

$b$

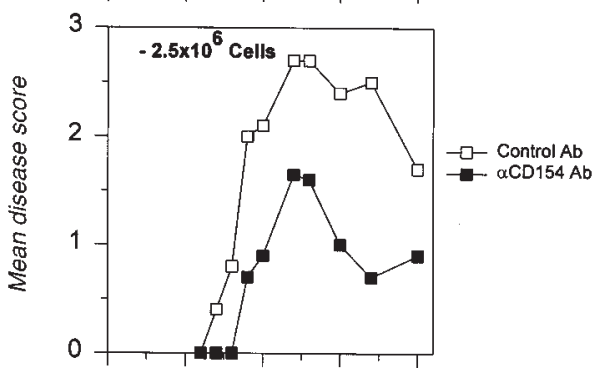

$C$

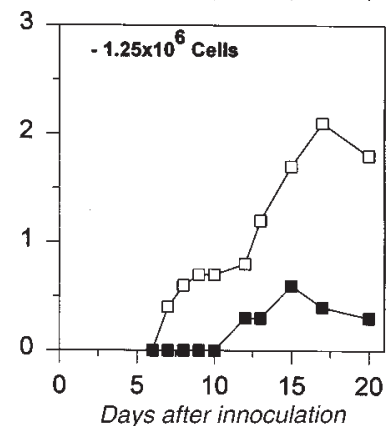

Figure 7

Anti-CD154 treatment of recipient mice diminishes the capacity of PLP139151-primed T cells to transfer R-EAE. Untreated female SJL/J mice were primed with PLP139-151/CFA, and draining lymph nodes were harvested on day 8 and cultured with $70 \mu \mathrm{g} / \mathrm{ml}$ PLP in vitro. After 4 days in culture, naive female SJL/J recipient mice were injected intravenously with $5 \times 10^{6}$ (a), $2.5 \times 10^{6}(\boldsymbol{b})$, or $1.25 \times 10^{6}(\mathbf{c})$ viable cells. Recipient mice were then treated with $250 \mu \mathrm{g}$ of control Ig (open boxes) or anti-CD154 (closed boxes) administered intraperitoneally three times a week. Mice were examined for disease severity and scored as described in Methods.

cytokines in the induction of resistance to experimental allergic encephalomyelitis. J. Neuroimmunol. 58:211-222.

10. Karpus, W.J., Pope, J.G., Peterson, J.D., Dal Canto, M.C., and Miller, S.D. 1995. Inhibition of Theiler's virus-mediated demyelination by peripheral immune tolerance induction. J. Immunol. 155:947-957.

11. Kuchroo, V.K., et al. 1995. B7-1 and B7-2 costimulatory molecules differentially activate the Th1/Th2 developmental pathways: application to autoimmune disease therapy. Cell. 80:707-718.

12. Perrin, P.J., et al. 1995. Role of B7:CD28/CTLA-4 in the induction of chronic relapsing experimental allergic encephalomyelitis. J. Immunol. 154:1481-1490.

13. Miller, S.D., et al. 1995. Blockade of CD28/B7-1 interaction prevents epitope spreading and clinical relapses of murine EAE. Immunity. 3:739-745.

14. Grewal, I.S., et al. 1996. Requirement for CD40 ligand in costimulation induction, T cell activation, and experimental allergic encephalomyelitis. Science. 273:1864-1867.

15. Gerritse, K., et al. 1996. CD40-CD40 ligand interactions in experimental allergic encephalomyelitis and multiple sclerosis. Proc. Natl. Acad. Sci. USA. 93:2499-2504

16. Griggs, N.D., et al. 1996. The relative contribution of the CD28 and gp39 costimulatory pathways in the clonal expansion and pathogenic acquisition of self-reactive T cells. J. Exp. Med. 183:801-810.

17. Carayanniotis, G., Masters, S.R., and Noelle, R.J. 1997. Suppression of murine thyroiditis via blockade of the CD40-CD40L interaction. Immunology. 90:421-426.

18. Mohan, C., Shi, Y., Laman, J.D., and Datta, S.K. 1995. Interaction between CD 40 and its ligand gp39 in the development of murine lupus 
nephritis. J. Immunol. 154:1470-1480.

19. Durie, F.H., et al. 1993. Prevention of collagen-induced arthritis with an antibody to gp39, the ligand for CD40. Science. 261:1328-1330.

20. Balasa, B., et al. 1997. CD40 ligand-CD40 interactions are necessary for the initiation of insulitis and diabetes in nonobese diabetic mice. $J$. Immunol. 159:4620-4627.

21. Mach, F., Schonbeck, U., Sukhova, G.K., Atkinson, E., and Libby, P. 1998 Reduction of atherosclerosis in mice by inhibition of CD40 signalling. Nature. 394:200-203.

22. Stout, R.D., and Suttles, J. 1996. The many roles of CD40 in cell-mediated inflammatory responses. Immunol. Today. 17:487-492.

23. Schultze, J.L., et al. 1997. CD40-activated human B cells: an alternative source of highly efficient antigen presenting cells to generate autologous antigen-specific $\mathrm{T}$ cells for adoptive immunotherapy. J. Clin. Invest. 100:2757-2765.

24. Van Kooten, C., and Banchereau, J. 1996. CD40-CD40 ligand: a multifunctional receptor-ligand pair. Adv. Immunol. 61:1-77.

25. Grewal, I.S., and Flavell, R.A. 1996. A central role of CD40 ligand in the regulation of CD4+ T-cell responses. Immunol. Today. 17:410-414.

26. Kelsall, B.L., Stuber, E., Neurath, M., and Strober, W. 1996. Interleukin12 production by dendritic cells. The role of CD40- CD40L interactions in Th1 T-cell responses. Ann. NY Acad. Sci. 795:116-126.

27. Dal Canto, M.C., and Lipton, H.L. 1975. Primary demyelination in Theiler's virus infection. An ultrastructural study. Lab. Invest. 33:626-637.

28. Stuber, E., Strober, W., and Neurath, M. 1996. Blocking the CD40L-CD40 interaction in vivo specifically prevents the priming of $\mathrm{T}$ helper 1 cells through the inhibition of interleukin 12 secretion. J. Exp. Med. 183:693-698.

29. Laman, J.D., Claassen, E., and Noelle, R.J. 1996. Functions of CD40 and its ligand, gp39 (CD40L). Crit. Rev. Immunol. 16:59-108.

30. Hancock, W.W., et al. 1996. Costimulatory function and expression of CD40 ligand, CD80, and CD86 in vascularized murine cardiac allograft rejection. Proc. Natl. Acad. Sci. USA. 93:13967-13972.

31. Early, G.S., Zhao, W., and Burns, C.M. 1996. Anti-CD40 ligand antibody treatment prevents the development of lupus-like nephritis in a subset of New Zealand black x New Zealand white mice. Response correlates with the absence of an anti-antibody response. J. Immunol. 157:3159-3164.

32. Hollenbaugh, D., et al. 1995. Expression of functional CD40 by vascular endothelial cells. J. Exp. Med. 182:33-40.

33. Dechanet, J., et al. 1997. CD40 ligand stimulates proinflammatory cytokine production by human endothelial cells. J. Immunol. 159:5640-5647.

34. Stout, R.D., Suttles, J., Xu, J., Grewal, I.S., and Flavell, R.A. 1996. Impaired T cell-mediated macrophage activation in CD40 ligand-deficient mice. J. Immunol. 156:8-11.

35. Malik, N., Greenfield, B.W., Wahl, A.F., and Kiener, P.A. 1996. Activation of human monocytes through CD40 induces matrix metalloproteinases. J. Immunol. 156:3952-3960.

36. Tian, L., Noelle, R.J., and Lawrence, D.A. 1995. Activated T cells enhance nitric oxide production by murine splenic macrophages through gp39 and LFA-1. Eur. J. Immunol. 25:306-309.

37. Karandikar, N.J., et al. 1998. Tissue-specific up-regulation of B7-1 expression and function during the course of murine relapsing experimental autoimmune encephalomyelitis. J. Immunol. 161:192-199.

38. Ranheim, E.A., and Kipps, T.J. 1993. Activated T cells induce expression of B7/BB1 on normal or leukemic B cells through a CD40-dependent signal. J. Exp. Med. 177:925-935.

39. Grewal, I.S., Xu, J., and Flavell, R.A. 1995. Impairment of antigen-specific T-cell priming in mice lacking CD40 ligand. Nature. 378:617-620.

40. Shu, U., et al. 1995. Activated T cells induce interleukin-12 production by monocytes via CD40-CD40 ligand interaction. Eur. J. Immunol. 25:1125-1128.

41. Cella, M., et al. 1996. Ligation of CD40 on dendritic cells triggers production of high levels of interleukin-12 and enhances T cell stimulatory capacity: T-T help via APC activation. J. Exp. Med. 184:747-752.

42. Garside, P., et al. 1998. Visualization of specific B and T lymphocyte interactions in the lymph node. Science. 281:96-99.

43. Samoilova, E.B., Horton, J.L., Zhang, H.D., and Chen, Y.H. 1997. CD40L blockade prevents autoimmune encephalomyelitis and hampers TH1 but not TH2 pathway of T cell differentiation. J. Mol. Med. 75:603-608.

44. Racke, M.K., et al. 1994. Cytokine-induced immune deviation as a therapy for inflammatory autoimmune disease. J. Exp. Med. 180:1961-1966. 